\title{
Parallel universes?
}

\section{An EU Commissioner has a meeting of minds with an antibiotech agitator.}

The EU's Environment Commissioner Stavros Dimas and long-time biotech critic Jeremy Rifkin of the Foundation on Economic Trends share some common sentiments. They both are passionate about the environment. They both purport to embrace new plant breeding technologies. And they're both pathologically averse to GM crops.

Earlier this year, at a meeting entitled 'Freedom of Choice Conference on GMO [genetically modified organism] Co-existence' Dimas declared: "GM products raise a whole new series of possible risks to the environment, notably potential longer-term effects that could impact on biodiversity." At the same conference, he claimed that "terminator technology" is on the market (it is not) and "small farmers are being put out of business by GMOs" (they are not). But he was almost gushing about what he terms "upgraded conventional varieties" created using marker-assisted selection (MAS) technology.

MAS is not rocket science-it is simply the use of genetic markers that segregate with valuable agronomic traits to rapidly identify crossbred seedlings that contain a specific trait of interest. Because you don't have to wait for a seedling to mature until it displays your trait of interest, MAS can cut development times of new commercial crop varieties in half. Coincidentally, in an article published in the Washington Post in July, Rifkin also waxes lyrical about the benefits of MAS, especially as a means of making "gene splicing and transgenic crops obsolete."

Like Rifkin, Dimas sees conventional "crop varieties upgraded through MAS as an alternative to GM crops." At the conference, he was particularly keen to promote upgraded crops as such. However, unlike Rifkin, Dimas seems unaware of a fatal flaw in his whole-hearted espousal of MAS.

Rifkin claims that MAS is of value when it is used as part of a broader, agro-ecological approach to farming, "one that integrates introduction of new crops with a proper regard for all the other environmental, economic and social factors that together determine the sustainability of farming." In other words, Rifkin, the antibiotech gadfly, acknowledges that plants can go bad even when they are not GM.

Commissioner Dimas, on the other hand, in his simple-minded, 'anything-but' approach to GM products, has not understood that a non-GM crop produced via MAS could be just as risky as a GM product; he hasn't grasped that upgraded crops with traits (e.g., herbicide resistance) like those of GM varieties could, by definition, carry the same risks as the GM variety. From an environmental risk assessment perspective, it is the new trait in the plant that is important, not the way in which a gene(s) associated with a trait ended up in the plant.

Unwittingly then, Dimas has disclosed for all to see, the glaring inequity and inadequacy of the current European regulatory framework. Because of its discriminatory and absolute emphasis on anything remotely GM to the exclusion of anything else, European regulation subjects a GM crop containing a trait analogous to that found in a conventional upgraded crop to intense environmental risk scrutiny, but leaves Dimas' upgraded crop with the same trait completely unregulated. The question that the Environment Commissioner should be asking himself is: what if that trait were harmful?

\section{Partnering with a difference}

\section{This month marks the inaugural EuroBiO meeting, a new type of biotech partnering event.}

$T^{\mathrm{h}}$ he licensing season is upon us, not that it ever really goes away. Europe's annual cycle of one-to-one meetings, open-house presentations and bioethanolic soirées begins this month with Techvision's BioPartnering Europe (BPE) in London, followed in November by BIOEurope in Düsseldorf, organized by EBD and the US Biotechnology Industry Organization (BIO). Sandwiched between them this year for the first time is a new event, EuroBiO, one major aim of which is to create an environment to promote early-stage partnering.

BioPartnering Europe and BIO-Europe have evolved over the years into meetings for the exchange of well-developed products or service lines (e.g., a phase 3 compound, candidates with human toxicity data and proof of concept in animals, or a neat assay method). In general, these are offerings for which risk has been reduced and reliability increased, and the value is relatively easy to assess. Deal discussions center less on price and more on the fit between business models, the prioritization of external projects and the alignment of expectations.

Partnering at EuroBiO, on the other hand, will be much more concerned with technology development and technology exchange as parties seek that combination of approaches that can provide solutions to industrially relevant problems, solutions that other firms will pay money for. The elements of the solutions are as likely to come from academia as from industry.
The other element that distinguishes EuroBiO as a partnering event is the presence of European Commission (EC) personnel. The EC's research funding staff will be at EuroBiO promoting corporate involvement in the new Framework Programme 7 (FP7). Their political masters at the European Parliament agreed in June that FP7 should be oriented much more to company needs, especially to the needs of small and medium-sized enterprises (SMEs). Such firms have participated in previous EC-funded research but the firm generally would have little say in designing the project and, as a consequence, might not benefit much from its outcome. In FP7, in contrast, companyled projects are the first priority. In the life sciences category, for instance, there are around 85 projects worth some $€ 200$ million in which SMEs are expected to take the lead. That means they set the agenda, include the collaborators they want and direct the project towards their own ends. Small companies will now get up to $75 \%$ of their costs from the EC, compared to $50 \%$ in previous Framework Programmes. And, of course, biotech firms can also compete for all the other elements of FP7.

So EuroBiO 2006 delivers on two fronts: it combines straight science technology partnering with the chance to get access to European research money by identifying and meeting potential partners for FP7 projects. 